Illinois Journal of Mathematics

Volume 45, Number 4, Winter 2001, Pages 1331-1345

S $0019-2082$

\title{
HARMONIC MAPS FROM FINSLER MANIFOLDS
}

\author{
XIAOHUAN MO
}

\begin{abstract}
A Finsler manifold is a Riemannian manifold without the quadratic restriction. In this paper we introduce the energy functional, the Euler-Lagrange operator, and the stress-energy tensor for a smooth map $\phi$ from a Finsler manifold to a Riemannian manifold. We show that $\phi$ is an extremal of the energy functional if and only if $\phi$ satisfies the corresponding Euler-Lagrange equation. We also characterize weak Landsberg manifolds in terms of harmonicity and horizontal conservativity. Using the representation of a tension field in terms of geodesic coefficients, we construct new examples of harmonic maps from Berwald manifolds which are neither Riemannian nor Minkowskian.
\end{abstract}

\section{Introduction}

Harmonic maps between Riemannian manifolds are mappings $\phi:(M, g) \rightarrow$ $(N, h)$ for which the "Dirichlet energy functional"

$$
\frac{1}{2} \int_{M}\|d \phi\|^{2} d v
$$

is extremal. Harmonic maps are solutions to an elliptic system of partial differential equations, which in general is non-linear. They are very important in both classical and modern differential geometry.

On the other hand, Riemannian manifolds are a special case of Finsler manifolds [9], namely Finsler manifolds with the quadratic restriction [8].

Let $\phi:(M, F) \rightarrow(N, h)$ be a smooth map from a Finsler manifold to a Riemannian manifold. In this paper we introduce a natural energy functional and the Euler-Lagrange operator of $\phi$. We show that $\phi$ is an extremal of the energy functional if and only if $\phi$ satisfies the corresponding Euler-Lagrange equation.

The weak Landsberg manifolds (see Definition 4.2) are special Finsler manifolds. They have constant volume of Finsler spheres and therefore satisfy the Gauss-Bonnet formula [4][13]. The weak Landsberg spaces have the following

Received October 24, 2000; received in final form June 4, 2001.

2000 Mathematics Subject Classification. Primary 53C60, 53B20. Secondary 54E40.

This work is supported by the National Natural Science Foundation of China (10171002) and the Foundation of School of Mathematical Sciences, Peking University. 
interesting geometric characterization: a Finsler manifold is of weak Landsberg type if and only if all projective spheres in the projective sphere bundle are minimal [6].

In this paper we introduce a notion of stress-energy tensor for maps from a Finsler manifold to a Riemannian manifold and characterize weak Landsberg manifolds in terms of harmonicity and horizontal conservativity. Here horizontal conservativity means that the stress-energy tensor is divergencefree with respect to the horizontal subbundle of the projective sphere bundle. We refer to [15] for the relation between horizontally conservative maps and harmonic morphisms.

\section{Preliminaries}

Let $M$ be a $C^{\infty} m$-dimensional manifold and $\widetilde{T M}=T M \backslash\{0\}$. A function $F: T M \rightarrow[0, \infty)$ is called a Finsler structure on $M$ if $F$ has the following properties:

(i) $F(t Y)=t F(Y)$ for all $t \in R^{+}$.

(ii) $F$ is $C^{\infty}$ on $\widetilde{T M}$.

(iii) For every non-zero $Y \in T_{x} M$, the induced quadratic form $g_{Y}$ given by

$$
g_{Y}(U, V):=\left.\frac{1}{2} \frac{\partial^{2}}{\partial s \partial t}\left(F^{2}(Y+s U+t V)\right)\right|_{s=t=0}
$$

is an inner product in $T_{x} M$.

A Finsler manifold is a $C^{\infty}$ manifold $M$ with a Finsler structure $F$.

Important examples of Finsler manifolds are Riemannian manifolds and Minkowski manifolds. Let $(M, F)$ be a Finsler manifold, $S M$ the projective sphere bundle of $M$, with canonical projection map $\pi: S M \rightarrow M$ given by $(x,[y]) \rightarrow x$, and let $S_{x} M:=\pi^{-1}(x)$ be the projective sphere at $x$. We denote the pull-backs of $T M$ and $T^{*} M$ by $\pi^{*} T M$ and $\pi^{*} T^{*} M$, respectively, and consider these as vector bundles (with $m$-dimensional fibres) over the $(2 m-1)$-dimensional base $S M$.

Given local coordinates $\left(x^{i}\right)$ on $M$, we can write any $y \in T_{x} M$ as $y^{i} \frac{\partial}{\partial x^{i}}$. This generates local coordinates $\left(x^{i} ; y^{i}\right)$ on $S M$.

At each point of $S M$, the fiber of $\pi^{*} T M$ has a basis $\left\{\frac{\partial}{\partial x^{i}}\right\}$. Hence $F$ inherits the Hilbert form, the fundamental tensor, and the Cartan tensor as follows:

$$
\begin{gathered}
\omega:=\frac{\partial F}{\partial y^{i}} d x^{i}, \quad g:=g_{i j}(x, y) d x^{i} \otimes d x^{j}, \quad g_{i j}=\left[\frac{1}{2} F^{2}\right]_{y^{i} y^{j}}, \\
A:=H_{i j k} d x^{i} \otimes d x^{j} \otimes d x^{k}, \quad H_{i j k}:=F \frac{\partial g_{i j}}{\partial y^{k}}
\end{gathered}
$$


where $1 \leq i, j, k \cdots \leq m=\operatorname{dim} M$. From the Cartan tensor one can construct the Cartan form by setting

$$
\eta=\sum_{i, j, k} H_{i j k} g^{j k} d x^{i}, \quad\left(g^{j k}\right)=\left(g_{j k}\right)^{-1} .
$$

This is a global section of $\pi^{*} T^{*} M \subset T^{*} S M$. We introduce a dual adapted orthonormal frame $\left\{e_{i}\right\}$ on the Riemannian vector bundle $\left(\pi^{*} T M, g\right)$ and a coframe $\left\{\omega_{i}\right\}$ with $\omega_{n}=\omega$. Putting $\omega_{i}=\sum v_{i j} d x^{j}$, we have $\operatorname{det}\left(v_{i j}\right)=$ $\sqrt{\operatorname{det}\left(g_{k l}\right)}$ and $g=\sum \omega_{i}^{2} \in \Gamma\left(\odot^{2} \pi^{*} T^{*} M\right)$.

Taking the exterior derivative of $\omega$ yields the Chern connection on $\pi^{*} T M$ described by an $m \times m$ matrix of 1-forms $\left(\omega_{i j}\right)$ on $S M$. These connection forms determine horizontal and vertical derivatives, Riemannian and Minkowski curvature, the Riemannian metric on $S M$ and its Riemannian connection [11]. Notice that

$$
\omega_{1} \wedge \cdots \wedge \omega_{m} \wedge \omega_{m 1} \wedge \cdots \wedge \omega_{m, m-1}
$$

is the volume form with respect to the Riemannian metric on $S M$. We denote it by $\Pi$.

The following lemmas will be used later.

LEMma 2.1. If $M$ is a compact Finsler manifold, then for any function $f: S M \rightarrow R$

$$
\int_{S M} f \Pi=\int_{M} d x \int_{S_{x} M} f \sqrt{\operatorname{det}\left(g_{i j}\right)} \chi
$$

where $d x=d x^{1} \wedge \cdots \wedge d x^{m}$ and

$$
\chi \equiv \omega_{m 1} \wedge \cdots \wedge \omega_{m, m-1} \quad \bmod \quad d x^{j} .
$$

In particular, if $M$ is Riemannian and $f$ is defined on $M$, then

$$
\int_{S M} f \Pi=\operatorname{Vol}\left(S^{m-1}\right) \int_{M} f d v
$$

where $\operatorname{Vol}\left(S^{m-1}\right)$ is the volume of standard $(m-1)$-dimensional sphere.

Proof. Obvious.

Set

$$
1 \leq \lambda, \mu, \tau, \ldots \leq m-1, \quad \bar{\lambda}=m+\lambda, \quad 1 \leq a, b, c, \ldots \leq 2 m-1 .
$$

The first structure equation for $(M, F)$ can be written as

$$
d \omega_{i}=\sum \omega_{j} \wedge \omega_{j i}, \quad \omega_{i j}+\omega_{j i}=-2 \sum A_{i j \lambda} \omega_{m \lambda},
$$

where $A_{i j k}=A\left(e_{i}, e_{j}, e_{k}\right)$. Taking the exterior derivative of (2.1) we see that the curvature 2-forms $\Omega_{i j}:=d \omega_{i j}-\sum \omega_{i k} \wedge \omega_{k j}$ can be expressed in the form

$$
\Omega_{i j}=\frac{1}{2} \sum R_{i j k l} \omega_{k} \wedge \omega_{l}+\sum P_{i j k \lambda} \omega_{k} \wedge \omega_{m \lambda},
$$


where $R_{i j k l}=-R_{i j l k}$. Set

$$
P_{\lambda \mu \nu}:=P_{m \lambda \mu \nu}
$$

$P_{\lambda \mu \nu}$ is called the Landsberg curvature [14]. From [4] we have

$$
P_{m \lambda \mu}=0, \quad \sum_{\lambda} P_{\lambda \lambda \mu}=-\sum_{\lambda} \dot{A}_{\lambda \lambda \mu},
$$

where the dot denotes the covariant derivative along the Hilbert form.

Denote the Riemannian metric on $S M$ by $G$ (cf. [11]). The divergence of a form $\Psi$ on $S M$ with respect to $G$ is defined by

$$
\operatorname{div} \Psi:=\sum_{a}\left(D_{\epsilon_{a}} \Psi\right)\left(, \epsilon_{a}\right)
$$

where $\left\{\epsilon_{a}\right\}$ is the dual basis of $\left\{\omega_{1}, \ldots, \omega_{m}, \omega_{m 1}, \ldots, \omega_{m, m-1}\right\}$ on $T(S M)$ and $D_{\epsilon_{a}}$ is the covariant derivative induced by $G$ along $\epsilon_{a}$.

LEMma 2.2 .

(i) For $S=\sum S_{i} \omega_{i} \in \Gamma\left(\pi^{*} T^{*} M\right)$, div $S=\sum S_{i \mid i}+\sum S_{\mu} P_{\lambda \lambda \mu}$.

(ii) For $T=\sum T_{i j} \omega_{i} \omega_{j} \in \Gamma\left(\odot^{2} \pi^{*} T^{*} M\right)$, $\operatorname{div} T\left(\epsilon_{i}\right)=\sum T_{i j \mid j}+\sum T_{i \mu} P_{\lambda \lambda \mu}$.

Proof. With the abbreviations

$$
\psi_{i}=\omega_{i}, \quad \psi_{\bar{\lambda}}=\omega_{m \lambda},
$$

denote the Levi-Civita connection with respect to $\left\{\psi_{a}\right\}$ by $\left\{\psi_{a b}\right\}$. Then (cf. [11])

$$
\psi_{i j} \equiv \omega_{j i} \quad \bmod \psi_{\bar{\lambda}}, \quad \psi_{i \bar{\lambda}} \equiv-\sum P_{i \lambda \mu} \psi_{\bar{\mu}} \quad \bmod \psi_{j},
$$

and

$$
\begin{aligned}
\operatorname{div} S & =\sum_{a}\left(D S_{a}\right)\left(\epsilon_{a}\right) \\
& =\sum\left(d S_{i}-\sum S_{j} \psi_{j i}\right)\left(\epsilon_{i}\right)-\sum S_{j} \psi_{j \bar{\lambda}}\left(\epsilon_{\bar{\lambda}}\right) \\
& =\sum\left(d S_{i}-\sum S_{j} \omega_{i j}\right)\left(\epsilon_{i}\right)-\sum S_{\mu}\left(-P_{\lambda \lambda \mu}\right) \\
& =\sum_{i} S_{i \mid i}+\sum S_{\mu} P_{\lambda \lambda \mu},
\end{aligned}
$$

where the covariant derivative of $S$ is defined by

$$
D S_{i}=d S_{i}-\sum S_{j} \omega_{i j}=\sum S_{i \mid j} \omega+\sum S_{i ; \lambda} \omega_{m \lambda} .
$$

This proves (i). 
Similarly, for $T$ we have

$$
\begin{aligned}
(\operatorname{div} T)\left(\epsilon_{i}\right) & =\sum\left(d T_{i b}-\sum T_{c b} \psi_{c i}-\sum T_{i c} \psi_{c b}\right)\left(\epsilon_{b}\right) \\
& =\sum\left(d T_{i j}-T_{k j} \omega_{i k}-\sum T_{i k} \omega_{j k}\right)\left(\epsilon_{j}\right)+\sum T_{i \mu} P_{\lambda \lambda \mu} \\
& =\sum T_{i j \mid j}+\sum T_{i \mu} P_{\lambda \lambda \mu},
\end{aligned}
$$

which proves (ii).

The energy density of a map $\phi:(M F) \rightarrow(N, h)$ from a Finsler manifold to a Riemannian manifold is the function $e(\phi): S M \rightarrow R_{\geq 0}$ defined by

$$
e(\phi)(x,[y])=\frac{1}{2} \sum_{j} h\left(\phi_{*} e_{j}, \phi_{*} e_{j}\right),
$$

where $\left\{e_{j}\right\}$ is the orthonormal basis with respect to $g$ (the fundamental tensor of $F)$ at $(x,[y])$.

If $\Omega$ is a compact domain in $M$, we use the canonical volume element $\Pi$ associated with $F$ to define the energy of $\phi:(\Omega, F) \rightarrow(N, h)$ by

$$
E(\phi, \Omega)=\frac{1}{c} \int_{S \Omega} e(\phi) \Pi,
$$

where $c:=\operatorname{Vol}\left(S^{m-1}\right)$ is the volume of the standard $(m-1)$-dimensional sphere and $S \Omega$ the projective sphere bundle of $\Omega$. If $M$ is compact, we write $E(\phi)=E(\phi, M)$.

REMARK. By Lemma 2.1, our notion of energy reduces to the usual notion of energy if $M$ is a compact Riemannian manifold.

A smooth map $\phi:(M, F) \rightarrow(N, h)$ from a Finsler manifold to a Riemannian manifold is said to be harmonic if it is an extremal of the restriction of $E$ on every compact subdomain of $(M, F)$.

\section{The first variation}

Let $(M, F)$ be a smooth Finsler manifold and $g$ the fundamental tensor of $F$. Let $(N, h)$ be a Riemannian manifold. Let $\phi:(M, F) \rightarrow(N, h)$ be a smooth map. Set

$$
h=\sum \theta_{\alpha}^{2} \in \Gamma\left(\odot^{2} T^{*} N\right), \quad 1 \leq \alpha, \beta, \gamma, \ldots \leq n .
$$

The first structure equation for $(N, h)$ is

$$
d \theta_{\alpha}=\sum \theta_{\beta} \wedge \theta_{\beta \alpha}, \quad \theta_{\alpha \beta}+\theta_{\beta \alpha}=0 .
$$

A vector field $v$ along $\phi$ determines a variation $\phi_{t}$ by $\phi_{t}(x)=\exp _{\phi(x)}[t v(x)]$, where $t \in I:=(-\varepsilon, \varepsilon)$ for some $\varepsilon>0$. Noting that

$$
\phi_{t}^{*} \theta_{\alpha} \in \Gamma\left(T^{*} M\right) \subset \Gamma\left(\pi^{*} T^{*} M\right),
$$


we put

$$
\phi_{t}^{*} \theta_{\alpha}=\sum a_{\alpha i} \omega_{i}
$$

where $a_{\alpha i}=a_{\alpha i}(t)$. It follows that

$$
\phi_{t}^{*}(h)=\phi_{t}^{*}\left(\sum \theta_{\alpha}^{2}\right)=\sum\left[\phi_{t}^{*} \theta_{\alpha}\right]^{2}=\sum a_{\alpha i} a_{\alpha j} \omega_{i} \omega_{j} .
$$

Since $\left\{e_{i}\right\}$ is the dual frame field of $\left\{\omega_{i}\right\}$, from (2.3) and (3.4) we obtain

$$
2 e\left(\phi_{t}\right)=\sum_{i}\left(\sum a_{\alpha j} a_{\alpha k} \omega_{j} \omega_{k}\right)\left(e_{i}, e_{i}\right)=\sum a_{\alpha i}^{2}
$$

If $v$ has compact support $\Omega \subset M$, then

$$
\left.c \cdot \frac{d}{d t} E\left(\phi_{t}, \Omega\right)\right|_{t=0}=\left.\int_{S M} \sum a_{\alpha i} \frac{\partial a_{\alpha i}}{\partial t}\right|_{t=0} \Pi .
$$

Define $\Phi: M \times I \rightarrow N$ by

$$
(x, t) \stackrel{\Phi}{\rightarrow} \phi_{t}(x)
$$

It is easy to see that

$$
\Phi^{*} \theta_{\alpha} \equiv \phi_{t}^{*} \theta_{\alpha}, \quad \Phi^{*} \theta_{\alpha \beta} \equiv \phi_{t}^{*} \theta_{\alpha \beta} \quad \bmod \quad d t
$$

Put

$$
\begin{aligned}
\Phi^{*} \theta_{\alpha} & =\phi_{t}^{*} \theta_{\alpha}+b_{\alpha} d t, \\
\Phi^{*} \theta_{\alpha \beta} & =\phi_{t}^{*} \theta_{\alpha \beta}+B_{\alpha \beta} d t .
\end{aligned}
$$

Then $\left.\sum b_{\alpha} v_{\alpha}\right|_{t=0}=b$ is the deformation vector field, where $\left\{v_{\alpha}\right\}$ is the dual frame field of $\left\{\theta_{\alpha}\right\}$, and $B_{\alpha \beta}$ satisfies

$$
B_{\alpha \beta}=-B_{\beta \alpha}
$$

Using (3.2), (3.3), (3.6), and (3.7), we obtain

$$
\begin{aligned}
d\left(\Phi^{*} \theta_{\alpha}\right) & =\Phi^{*}\left(d \theta_{\alpha}\right) \\
& =\Phi^{*}\left(\sum \theta_{\beta} \wedge \theta_{\beta \alpha}\right) \\
& =\sum \Phi^{*} \theta_{\beta} \wedge \Phi^{*} \theta_{\beta \alpha} \\
& =\sum\left(\sum_{i} a_{\beta i} \omega_{i}+b_{\beta} d t\right) \wedge\left(\phi_{t}^{*} \theta_{\beta \alpha}+B_{\beta \alpha} d t\right) .
\end{aligned}
$$


On the other hand, from (3.3) and (3.6), we have

$$
\begin{aligned}
d\left(\Phi^{*} \theta_{\alpha}\right) & =d\left[\sum a_{\alpha i} \omega_{i}+b_{\alpha} d t\right] \\
& =\sum\left(d a_{\alpha i}\right) \wedge \omega_{i}+\sum a_{\alpha i} d \omega_{i}+d b_{\alpha} \wedge d t \\
& =\sum\left(d_{S M} a_{\alpha i}+\frac{\partial a_{\alpha i}}{\partial t} d t\right) \wedge \omega_{i}+\sum a_{\alpha i} d \omega_{i}+d_{S M} b_{\alpha} \wedge d t
\end{aligned}
$$

Comparing the coefficients of $d t$ in (3.9) and (3.10), we obtain

$$
\sum \frac{\partial a_{\alpha i}}{\partial t} \omega_{i}-d_{S M} b_{\alpha}=\sum_{\beta}\left(b_{\beta} \phi_{t}^{*} \theta_{\beta \alpha}-\sum_{i} B_{\beta \alpha} a_{\beta i} \omega_{i}\right) .
$$

Define the covariant derivative of $\left\{b_{\alpha}\right\}$ by

$$
\begin{aligned}
D b_{\alpha} & =d_{S M} b_{\alpha}-\sum_{\beta} b_{\beta} \phi_{t}^{*} \theta_{\alpha \beta} \\
& =\sum b_{\alpha \mid i} \omega_{i}+\sum b_{\alpha ; \lambda} \omega_{m \lambda} .
\end{aligned}
$$

Substituting (3.12) into (3.11) we obtain

$$
\frac{\partial a_{\alpha i}}{\partial t}=b_{\alpha \mid i}-\sum B_{\beta \alpha} a_{\beta i}, \quad b_{\alpha ; \lambda}=0 .
$$

From (3.5), (3.8), and (3.13) we have

$$
\begin{aligned}
\left.c \cdot \frac{d}{d t} E\left(\phi_{t}\right)\right|_{t=0} & =\int \sum a_{\alpha i}\left(b_{\alpha \mid i}-\sum B_{\beta \alpha} a_{\beta i}\right) \Pi \\
& =\int\left(\sum a_{\alpha i} b_{\alpha \mid i}-\sum a_{\alpha i} B_{\beta \alpha} a_{\beta i}\right) \Pi \\
& =\int \sum_{i}\left(\sum a_{\alpha i} b_{\alpha}\right)_{\mid i} \Pi-\int \sum a_{\alpha i \mid i} b_{\alpha} \Pi,
\end{aligned}
$$

where

$$
a_{\alpha i \mid j}:=\left[d a_{\alpha i}-\sum a_{\alpha k} \omega_{i k}-\sum a_{\beta i} \phi^{*} \theta_{\alpha \beta}\right]\left(e_{j}\right)
$$

and

$$
\sum_{i}\left(\sum a_{\alpha i} b_{\alpha}\right) \omega_{i}=\langle d \phi, b\rangle
$$

is a global section on the dual Finsler bundle $\pi^{*} T^{*} M$. Using (2.2) and Lemma 2.2 we get

$$
\begin{aligned}
\left.c \cdot \frac{d}{d t} E\left(\phi_{t}\right)\right|_{t=0} & =\int_{S M} \operatorname{div}\langle d \phi, b\rangle \Pi-\int_{S M}\langle\tau(\phi), b\rangle \Pi \\
& =-\int_{S M}\langle\tau(\phi), b\rangle \Pi,
\end{aligned}
$$


where

$$
\tau(\phi):=-\langle d \phi, \dot{\eta}\rangle+\operatorname{Tr} D d \phi \in \Gamma\left((\phi \circ \pi)^{*} T N\right)
$$

and $\eta$ (resp. $D d \phi$ ) denotes the Cartan form (resp. the second fundamental form) of $\phi$. The field $\tau(\phi)$ is called the tension field of $\phi$.

THEOREM 3.1. Let $\phi$ be a smooth map from a Finsler manifold $M$ to a Riemannian manifold $N$. Then $\phi$ is harmonic if and only if it has vanishing tension field.

Let us now express the tension field in local coordinates $\left(x^{i}\right)$ on $M$ and $\left(u^{\alpha}\right)$ on $N$. We denote by $g_{i j}$ and ${ }^{M} \Gamma_{j k}^{i}$ the components of the fundamental tensor and the Christoffel symbols of the Chern connection on $(M, F)$, and by $h_{\alpha \beta}$ and ${ }^{N} \Gamma_{\beta \gamma}^{\alpha}$ the corresponding objects on $(N, h)$. Note that ${ }^{N} \Gamma_{\beta \gamma}^{\alpha}$ are just the Christoffel symbols of Levi-Civita on $N$ because $h_{\alpha \beta}$ are Riemannian.

Let $D$ denote the covariant differentiation (of sections of tensor products of $\pi^{*} T M$ and $\left.\pi^{*} T^{*} M\right)$ on $S M$ with respective to the Chern connection. Then, by $(2.46)$ and $(2.47 \mathrm{a})$ in [3], we have

$$
D \frac{\partial}{\partial x^{k}}={ }^{M} \Gamma_{k l}^{i} d x^{l} \otimes \frac{\partial}{\partial x^{i}},
$$

where

$$
\begin{gathered}
{ }^{M} \Gamma_{k l}^{i}=g^{i j M} \Gamma_{j k l}, \\
{ }^{M} \Gamma_{j k l}=\frac{1}{2}\left(\frac{\partial g_{j k}}{\partial x^{l}}-\frac{\partial g_{k l}}{\partial x^{j}}+\frac{\partial g_{l j}}{\partial x^{k}}\right)+\frac{1}{2}\left(M_{j k l}-M_{k l j}+M_{l j k}\right), \\
M_{i j k}=-\frac{\partial g_{i j}}{\partial y^{l}} \frac{\partial G^{l}}{\partial y^{k}},
\end{gathered}
$$

and $G^{l}$ are the geodesic coefficients of $(M, F)$ (cf. [12]). Using the Leibniz rule, we obtain (cf. [5, p. 41])

$$
D d x^{i}=-{ }^{M} \Gamma_{k l}^{i} d x^{k} \otimes d x^{l} .
$$

Suppose that $\phi:(M, F) \rightarrow(N, h)$ is a smooth map. Locally, we can write $\phi=\left(\phi^{\alpha}\right)$ where each $\phi^{\alpha}$ is a smooth function defined an open subset in $M$. Let $D$ denote the covariant differentiation on $\pi^{*} T^{*} M \otimes(\phi \circ \pi)^{*} T N$. Then (cf. [10])

$$
\begin{aligned}
D_{\partial / \partial x^{i}}(d \phi) & =D_{\partial / \partial x^{i}}\left(\phi_{j}^{\alpha} d x^{j} \frac{\partial}{\partial u^{\alpha}}\right) \\
& =\phi_{i j}^{\alpha} d x^{j} \frac{\partial}{\partial u^{\alpha}}+\phi_{j}^{\alpha} D_{\partial / \partial x^{i}}^{\pi^{*} T^{*} M} d x^{j} \frac{\partial}{\partial u^{\alpha}}+\phi_{j}^{\alpha} d x^{j} D_{\partial / \partial x^{i}}^{(\phi \circ \pi)^{*} T N} \frac{\partial}{\partial u^{\alpha}},
\end{aligned}
$$

where

$$
\phi_{i}^{\alpha}=\frac{\partial \phi^{\alpha}}{\partial x^{i}}, \quad \phi_{i j}^{\alpha}=\frac{\partial^{2} \phi^{\alpha}}{\partial x^{i} \partial x^{j}} .
$$


Now

$$
D_{\partial / \partial x^{i}}^{\pi^{*} T^{*} M} d x^{j}=-{ }^{M} \Gamma_{k i}^{j} d x^{k}
$$

and

so that

$$
D_{\partial / \partial x^{i}}^{\pi^{*} \phi^{*} T N} \frac{\partial}{\partial u^{\alpha}}=\phi_{i}^{\beta N} \Gamma_{\alpha \beta}^{\gamma} \frac{\partial}{\partial u^{\gamma}},
$$

$$
D_{\partial / \partial x^{i}}(d \phi)=\left(\phi_{i j}^{\alpha}-{ }^{M} \Gamma_{i j}^{k} \phi_{k}^{\alpha}+{ }^{N} \Gamma_{\beta \gamma}^{\alpha} \phi_{i}^{\beta} \phi_{j}^{\gamma}\right) d x^{j} \frac{\partial}{\partial u^{\alpha}},
$$

where we have used the fact that (cf. [3])

$$
{ }^{M} \Gamma_{i j}^{k}={ }^{M} \Gamma_{j i}^{k}
$$

It follows that the components of the second fundamental form $D d \phi$ satisfy

$$
(D d \phi)_{i j}^{\alpha}=\phi_{i j}^{\alpha}-{ }^{M} \Gamma_{i j}^{k} \phi_{k}^{\alpha}+{ }^{N} \Gamma_{\beta \gamma}^{\alpha} \phi_{i}^{\beta} \phi_{j}^{\gamma} .
$$

Now consider a smooth function $f$ defined on an open subset in $M$. Set

$$
f_{j}=\frac{\partial f}{\partial x^{j}}, \quad f_{i j}=\frac{\partial f_{j}}{\partial x^{i}},
$$

and

$$
D_{\partial / \partial x^{i}}(d f)=(D d f)_{i j} d x^{j}
$$

Then

$$
\begin{gathered}
d f=f_{j} d x^{j} \\
(D d f)_{i j}=(D d f)\left(\frac{\partial}{\partial x^{i}}, \frac{\partial}{\partial x^{j}}\right)=f_{i j}-{ }^{M} \Gamma_{i j}^{k} f_{k} .
\end{gathered}
$$

Thus (3.14) reduces to

$$
\begin{aligned}
\tau(f) & =-\langle d f, \dot{\eta}\rangle+\operatorname{Tr} D d f \\
& =g^{i j}\left[f_{i j}-{ }^{M} \Gamma_{i j}^{k} f_{k}-\xi_{i} f_{j}\right],
\end{aligned}
$$

where

$$
\xi_{j}=\dot{\eta}\left(\frac{\partial}{\partial x^{j}}\right) .
$$

Suppose that $\phi:(M, F) \rightarrow(N, h)$ is a smooth map. By (3.19) we have

$$
\tau\left(\phi^{\alpha}\right)=g^{i j}\left[\phi_{i j}^{\alpha}-{ }^{M} \Gamma_{i j}^{k} \phi_{k}^{\alpha}-\xi_{i} \phi_{j}^{\alpha}\right] .
$$

Hence the tension field of $\phi$ is

$$
\begin{aligned}
\tau_{\phi}^{\alpha} & =d u^{\alpha}(-\langle d \phi, \dot{\eta}\rangle+\operatorname{Tr} D d \phi) \\
& =g^{i j}\left[-\xi_{i} \phi_{j}^{\alpha}+\phi_{i j}^{\alpha}-{ }^{M} \Gamma_{i j}^{k} \phi_{k}^{\alpha}+{ }^{N} \Gamma_{\beta \gamma}^{\alpha} \phi_{i}^{\beta} \phi_{j}^{\gamma}\right] \\
& =\tau\left(\phi^{\alpha}\right)+g^{i j N} \Gamma_{\beta \gamma}^{\alpha} \phi_{i}^{\beta} \phi_{j}^{\gamma} .
\end{aligned}
$$


A direct calculation using (2.2), (3.15), (3.16), and (3.17) yields (cf. [5, (3.3.3)])

$$
\xi_{i}=-y^{j} \frac{\partial^{M} \Gamma_{j k}^{k}}{\partial y^{i}}
$$

and

$$
{ }^{M} \Gamma_{k i}^{i}=\left(\frac{\partial}{\partial x^{k}}-\frac{\partial G^{i}}{\partial y^{k}} \frac{\partial}{\partial y^{i}}\right) \log \sqrt{\operatorname{det}\left(g_{j l}\right)} .
$$

\section{The stress-energy tensor}

Let $\phi:(M, F) \rightarrow(N, h)$ be a smooth map from a Finsler manifold $(M, F)$ to a Riemannian manifold $(N, h)$. The stress-energy tensor $S_{\phi}$ is a tensor on $S M$ defined by

$$
S_{\phi}:=e(\phi) g-\phi^{*} h,
$$

where $e(\phi)$ (resp. $g$ ) denotes the energy density (resp. the fundamental tensor) of $\phi$ and $\phi^{*} h$ denotes the pull back of the tensor $h$ to a tensor on $S M$. We say that $S_{\phi}$ is horizontally divergence-free if $\sum_{i=1}^{m}\left(D_{\epsilon_{i}} S_{\phi}\right)\left(\epsilon_{i}, Y\right)=0$ for all $Y \in H_{p}$, where $\left\{\epsilon_{i}\right\}$ is any orthonormal basis for the horizontal space $H_{p}$ and $H_{p}:=\left\{X \in T_{p} S M, \omega_{m \lambda}(X)\right\}=0$ (cf. [11]).

Let $\phi:(M, F) \rightarrow(N, h)$ be a smooth map. Define on $M$

$$
\left\langle\theta_{\alpha}, d \phi\right\rangle=\sum a_{\alpha i} \omega_{i},
$$

where $\left\{\theta_{\alpha}\right\}$ is an orthonormal coframe of $h$. Then

$$
\begin{aligned}
d\left(\sum_{i} a_{\alpha i} \omega_{i}\right) & =d\left(\phi^{*} \theta_{\alpha}\right) \\
& =\phi^{*} d \theta_{\alpha} \\
& =\phi^{*}\left(\sum \theta_{\beta} \wedge \theta_{\beta \alpha}\right) \\
& =\sum \phi^{*} \theta_{\beta} \wedge \phi^{*} \theta_{\beta \alpha},
\end{aligned}
$$

by (3.2). Consider (4.1) as a two-form defined on the projective sphere bundle $S M$. We have

$$
\begin{aligned}
d\left(\sum_{i} a_{\alpha i} \omega_{i}\right) & =\sum d a_{\alpha i} \wedge \omega_{i}+\sum a_{\alpha i} d \omega_{i} \\
& =\sum d a_{\alpha i} \wedge \omega_{i}+\sum a_{\alpha i} \omega_{j} \wedge \omega_{j i} \\
& =\sum a_{\beta i} \omega_{i} \wedge \phi^{*} \theta_{\beta \alpha} .
\end{aligned}
$$

It follows that

$$
\sum_{i} D a_{\alpha i} \wedge \omega_{i}=0
$$


where

$$
\begin{aligned}
D a_{\alpha i} & :=d a_{\alpha i}-\sum a_{\alpha j} \omega_{i j}+\sum a_{\beta i} \phi^{*} \theta_{\beta \alpha} \\
& :=\sum a_{\alpha i \mid j} \omega_{j}+\sum a_{\alpha i ; \lambda} \omega_{m \lambda} .
\end{aligned}
$$

Substituting (4.3) into (4.2) yields the following result.

Proposition 4.1. The second fundamental form of $\phi:(M, F) \rightarrow(N, g)$ satisfies $a_{\alpha i \mid j}=a_{\alpha j \mid i}$ and $a_{\alpha i ; \lambda}=0$.

Denote the stress-energy $S_{\phi}$ of $\phi$ by

$$
S_{\phi}=\sum S_{i j} \omega_{i} \otimes \omega_{j}
$$

Then

$$
S_{i j}=e(\phi) \delta_{i j}-\sum a_{\alpha i} a_{\alpha j}
$$

where $e(\phi)$ is the energy density of $\phi$. Then the horizontal divergence of $S_{\phi}$ is

$$
\begin{aligned}
\operatorname{div}_{H} S_{\phi}= & \sum_{i j j} S_{i} \\
= & \sum_{i}\left(\sum_{i j \mid j}+\sum S_{i \mu} P_{\lambda \lambda \mu}\right) \omega_{i} \\
= & \sum_{i}\left\{\sum_{j}\left[e(\phi) \delta_{i j}-\sum a_{\alpha i} a_{\alpha j}\right]_{\mid j}\right. \\
& \left.+\sum_{i}\left[e(\phi) \delta_{i \mu}-\sum a_{\alpha i} a_{\alpha \mu}\right] P_{\lambda \lambda \mu}\right\} \omega_{i} \\
= & \sum_{i}\left[e(\phi)_{\mid i}-\sum a_{\alpha i \mid j} a_{\alpha j}-\sum a_{\alpha i} a_{\alpha j \mid j}\right. \\
& \left.+\sum_{i} e(\phi) P_{\lambda \lambda i}-\sum a_{\alpha i} a_{\alpha \mu} P_{\lambda \lambda \mu}\right] \omega_{i} \\
= & -\sum a_{\alpha j} a_{\alpha j \mid i}-\sum a_{\alpha i \mid j} a_{\alpha j} \\
& \left.\left.\quad+\sum a_{\alpha i} a_{\alpha j \mid j}+\sum e(\phi) P_{\lambda \lambda i}-\sum a_{\alpha i} a_{\alpha \mu} P_{\lambda \lambda \mu}\right] \omega_{i} P_{\lambda \lambda \mu}\right] \omega_{i}+e(\phi) \sum P_{\lambda \lambda \mu} \omega_{\mu} \\
= & \left.-\sum \tau(\phi), d \phi\right\rangle-e(\phi) \dot{\eta},
\end{aligned}
$$

where $\dot{\eta}$ denotes the covariant derivative of the Cartan form along the Hilbert form, and where we have used (2.2) and (ii) of Lemma 2.2. 
Definition 4.2. A Finsler manifold is said to be of weak Landsberg type if $\dot{\eta}=0$.

The following theorems are immediate consequences of (4.5).

TheOREM 4.3. Let $\phi:(M, F) \rightarrow(N, h)$ be a non-constant harmonic map from a Finsler manifold to a Riemannian manifold. Then $S_{\phi}$ is horizontally divergence-free if and only if $(M, F)$ is of weak Landsberg type.

Combining this with Shen's theorem ([6], [11]) (see the Introduction) we obtain the following Wood type result (cf. [15, Theorem 2.9]).

ThEOREM 4.4. Let $\phi:(M, F) \rightarrow(N, h)$ be a submersion from a Finsler manifold to a Riemannian manifold. Then any two of the following conditions imply the third condition:

(i) $\phi$ is harmonic;

(ii) $S_{\phi}$ is horizontally divergence-free;

(iii) $\pi: S M \rightarrow M$ has minimal fibers.

\section{Harmonicity of the identity map}

In this section we present the harmonic equation of the identity map from a Finsler manifold to a Riemannian manifold in terms of their geodesic coefficients, and we construct harmonic maps from Berwald manifolds which are neither Riemannian nor Minkowskian to Riemannian manifolds.

Let $I:(M, F) \rightarrow(M, h)$ be the identity map from a Finsler manifold $(M, F)$ to a Riemannian manifold $(M, h)$. As usual we put

$$
I=\left(I^{i}\right): U(\subset M) \rightarrow R,
$$

where locally $I^{i}\left(x^{1}, \ldots, x^{m}\right)=x^{i}$. It follows that

$$
I_{j}^{i}=\frac{\partial I^{i}}{\partial x^{j}}=\delta_{j}^{i}, \quad I_{j k}^{i}=0 .
$$

Using (3.21) and (3.22) we have

$$
\tau\left(I^{k}\right)=-g^{i j F} \Gamma_{i j}^{k}-g^{k j} \xi_{j},
$$

and hence

$$
\tau_{I}^{k}=g^{i j}\left[{ }^{h} \Gamma_{i j}^{k}-{ }^{F} \Gamma_{i j}^{k}\right]-g^{k j} \xi_{j} .
$$

Denote the geodesic coefficients of $(M, F)$ and $(M, h)$ by ${ }^{F} G^{i}$ and ${ }^{h} G^{i}$, respectively. By $[5,(3.8 .3)]$ we have

$$
\frac{1}{2}\left({ }^{F} G^{i}\right)_{y^{j} y^{k}}={ }^{F} \Gamma_{j k}^{i}+\dot{H}^{i}{ }_{j k},
$$


where $\dot{H}^{i}{ }_{j k}$ is the covariant derivative of the Cartan tensor along the Hilbert form. It follows that

$$
\begin{aligned}
g^{i j F} \Gamma_{i j}^{k}+g^{k i} \xi_{i} & =g^{i j}\left[\frac{1}{2}\left({ }^{F} G^{k}\right)_{y^{i} y^{j}}-\dot{H}_{i j}^{k}\right]+g^{k i} \dot{H}_{i} \\
& =\frac{1}{2} g^{i j}\left({ }^{F} G^{k}\right)_{y^{i} y^{j}}-\dot{H}^{k}+\dot{H^{k}}=\frac{1}{2} g^{i j}\left({ }^{F} G^{k}\right)_{y^{i} y^{j}}
\end{aligned}
$$

where in the second step we used $[5,(2.5 .11)]$. Similarly, for the Riemannian metric $h$ we have

$$
\frac{1}{2}\left({ }^{h} G^{i}\right)_{y^{j} y^{k}}={ }^{h} \Gamma_{j k}^{i} .
$$

Substituting (5.2) and (5.3) into the harmonic equation (5.1) gives

$$
\tau_{I}^{k}=\frac{1}{2} g^{i j}\left({ }^{h} G^{k}-{ }^{F} G^{k}\right)_{y^{i} y^{j}} .
$$

Thus we have the following result.

Proposition 5.1. Let $(M, h)$ be a flat Riemannian space. Then, for any local Minkowski structure $F$ on $M$, the identity map

$$
I:(M, F) \rightarrow(M, h)
$$

is harmonic.

Proof. By $[12,(3.23)]$ we have

$$
{ }^{F} G^{j}=\frac{1}{2} \sum_{i, k, l} g^{j l}\left[2 \frac{\partial g_{i l}}{\partial x^{k}}-\frac{\partial g_{i k}}{\partial x^{l}}\right] y^{i} y^{k} .
$$

On the other hand, $F$ is local Minkowskian if and only if

$$
g_{i j}(x, y)=g_{i j}(y) .
$$

The conclusion is now immediate from (5.3)-(5.5).

Definition 5.2. A Finsler manifold $(M, F)$ is said to be of

(i) Randers type if $F=\alpha+\beta$, where $\alpha$ is a Riemannian metric on $M$ and $\beta=\beta_{i} d x^{i}$ is a 1 -form;

(ii) Berwald type if $F$ has vanishing Minkowski curvature, i.e., if $P_{i j k \lambda}=0$ for all $i, j, k, \lambda$ (cf. [7]).

It is easy to see that a Randers manifold $(M, \alpha+\beta)$ is a Berwald manifold if and only if $\beta_{i \mid j}=0$, where $\beta_{i \mid j}$ is the covariant derivative of $\beta$ with respect to the Riemannian metric $\alpha$, that is, if the 1 -form $\beta$ is parallel with respect to $\alpha$. In this case, the Randers metric $\alpha+\beta$ and Riemannian metric $\alpha$ have same geodesic coefficients (cf. $[5,11.3 .11])$. Combining this with (5.4) we obtain: 
Proposition 5.3. Let $(M, \alpha+\beta)$ be a Randers manifold. If $\beta$ is parallel with respect to the Riemannian metric $\alpha$, then the identity

$$
I:(M, \alpha+\beta) \rightarrow(M, \alpha)
$$

is harmonic.

Antonelli, Ingarden, and Matsumoto [1] showed that Berwald manifolds which are neither Riemannian nor Minkowskian can be constructed using certain Randers metrics. In view of this, our results give examples of harmonic maps from Berwald manifolds which are neither Riemannian nor Minkowskian.

Acknowledgements. I would like to express my gratitude to Professor S. S. Chern for his continued encouragement during the preparation of this work, Professor E. Loubeau for his valuable comments, and the referee for his careful reading of the manuscript and very helpful suggestions. The final version of this paper was written during a visit to the Max-Planck Institute for Mathematics, Bonn; I would like to thank Professor Y. Manin and the Institute for their hospitality.

\section{REFERENCES}

[1] P. L. Antonelli, R. S. Ingarden, and M. Matsumoto, The theory of sprays and Finsler spaces with applications in physics and biology, Kluwer Academic Publishers, Dordrecht, 1993.

[2] D. Bao and B. Lackey, A Hodge decomposition theorem for Finsler spaces, C. R. Acad. Sci. Paris 323 (1996), 51-56.

[3] D. Bao and S. S. Chern, A notable connection in Finsler geometry, Houston J. Math. 19 (1993), 135-180.

[4] _ A note on the Gauss-Bonnet theorem for Finsler spaces, Ann. of Math. 143 (1996), 943-957.

[5] D. Bao, S. S. Chern, and Z. Shen, An introduction to Riemann-Finsler geometry, Graduate Texts in Math., vol. 200, Springer-Verlag, New York, 2000.

[6] D. Bao and Z. Shen, On the volume of unit tangent spaces in a Finsler manifold, Results Math. 26 (1994), 1-17.

[7] S. S. Chern, On Finsler geometry, C. R. Acad. Sci. Paris 314 (1992), 757-761.

[8] _ Finsler geometry is just Riemannian geometry without the quadratic restriction, Notices Amer. Math. Soc. 43 (1996), 959-963.

[9] _ Riemannian geometry as a special case of Finsler geometry, Finsler geometry (Seattle, WA, 1995), Contemp. Math., vol. 196, Amer. Math. Soc., Providence, RI, 1996, pp. 51-58.

[10] J. Eells and L. Lemaire, Select topics of harmonic maps, CBMS Regional Conf. Ser. in Math., vol. 50, Amer. Math. Soc., Providence, RI, 1983.

[11] X. Mo, Characterization and structure of Finsler spaces with constant flag curvature, Sci. China 41 (1998), 910-917.

[12] Z. Shen, Differential geometry of spray and Finsler spaces, Kluwer Academic Publishers, Dordrecht, 2001.

[13] _ Some formulas of Gauss-Bonnet-Chern type in Riemann-Finsler geometry, J. Reine Angew. Math. 475 (1996), 149-165. 
[14] _ Geometric meanings of curvatures in Finsler geometry, Proc. 20th Winter School "Geometry and Physics" (Srni, 2000), Rend. Circ. Mat. Palermo (2) Suppl. 66 (2001), 165-178.

[15] J. C. Wood, Harmonic morphisms, foliations and Gauss maps, Complex differential geometry and nonlinear differential equations (Brunswick, Maine, 1984), Contemp. Math., vol. 49, Amer. Math. Soc., Providence, RI, 1986, pp. 145-183.

LMam, School of Mathematical Sciences, Peking University, Beijing 100871, CHINA

E-mail address: moxh@pku.edu.cn 